\title{
Procedimentos Diagnósticos e Terapêuticos: Processo de Inserção de Habilidades e Competências Médicas no Currículo do Curso de Medicina da Anhanguera-Uniderp
}

\author{
Diagnostic and Therapeutic Procedures: \\ Defining Skills and Competencies in the \\ Medical Curriculum of Anhanguera-Uniderp \\ University
}

\author{
André Luís Alonso Domingos \\ Antônio Carlos Carbonaro Salles ${ }^{I}$ \\ Alexandre de Abreu Lima \\ Gabriel Costa Almeida \\ Marcia Maria Silva ${ }^{I}$ \\ Sandra Christo dos Santos ${ }^{I}$
}

\section{PALAVRAS-CHAVE}

- Procedimentos Médicos;

- Competências;

- Curriculum Médico;

- Educação Médica.

\section{KEYWORDS}

- Medical Procedures;

- Competencies;

- Medical Curriculum;

- Medical Education.

Recebido em: 29/06/2013

Reencaminhado em: 17/06/2014

Aprovado em: 31/08/2014

REVISTA BRASILEIRA DE EDUCAÇ̃̃o MÉDICA

\section{RESUMO}

Introdução: Há pouca discussão na literatura sobre quais procedimentos médicos o estudante de Medicina deve ser capaz de realizar no final do curso de graduação e em qual momento do currículo escolar eles devem ser inseridos. Metodologia: Uma comissão formada por especialistas de diversas áreas organizou uma lista de possíveis procedimentos médicos diagnósticos e terapêuticos, sugerida por estudos e debates do grupo e que estivessem de acordo com as Diretrizes Curriculares Nacionais de 2001. Após consenso do grupo, mediante aprovação de mais de $75 \%$ dos integrantes da comissão, os procedimentos que de fato deveriam integrar o currículo foram definidos. Resultados: Mais de 50 procedimentos médicos foram definidos, de acordo com o nível de complexidade esperada para o aluno de graduação. Após esta definição, tais procedimentos foram distribuídos na matriz curricular e divididos didaticamente em momentos de aprendizagem de domínio cognitivo e domínio motor. Conclusão: O presente estudo apresentou uma proposta de definição das competências e habilidades relativas aos procedimentos médicos a serem alcançadas pelos estudantes de Medicina de graduação em nossa instituição e teve como objetivo sistematizar a sua distribuição na matriz curricular.

\section{ABSTRACT}

Background: There is little discussion in the literature about which medical procedures students must be able to perform at the end of a medical course and at which point of the school curriculum they must be inserted. Methodology: A commission of specialists from different medical areas organized a list of possible diagnostic and therapeutic medical procedures, suggested by studies and group debates and followed the Brazilian curricular guidelines of 2001. Following group consensus with approval by over $75 \%$ of the commission members, the procedures that should actually be part of the school curriculum were defined. Results: Over 50 medical procedures were grouped according to the level of complexity expected of the undergraduate student at the end of medical course. Once these were defined, they were distributed in the curricular grid and split into distinct learning times of the cognitive and motor domains. Conclusion: This study presented a proposal to define the competencies and skills relative to medical procedures to be attained by the undergraduate medicine students at our institution, with the objective of systematizing their distribution in the curricular matrix. 


\section{INTRODUÇÃO}

As habilidades clínicas, o conhecimento científico e o comportamento ético/moral fundamentam as bases para o desenvolvimento de competências médicas ${ }^{1}$. Entre as competências a serem alcançadas estão a realização da entrevista médica, o exame físico e raciocínio clínico e a capacidade de realizar procedimentos diagnósticos e terapêuticos. A formação do médico deve contemplar o aprendizado de procedimentos clínicos e cirúrgicos fundamentais para a assistência ambulatorial e de urgência de acordo com as Diretrizes Curriculares para Cursos de Medicina no Brasil ${ }^{2}$. No entanto, os indicativos apontados pelas Diretrizes Curriculares geram espaço para discussão e dúvidas sobre quais procedimentos devem integrar o currículo dos cursos de graduação em Medicina e em que etapa do curso de graduação devem ser desenvolvidos.

A Association of American Medical Colleges (AAMC) definiu alguns procedimentos básicos como fundamentais para o aprendizado durante a graduação em Medicina ${ }^{3}$, porém estudos posteriores evidenciaram que apenas metade das escolas médicas americanas ensinava e avaliava adequadamente a execução dos procedimentos médicos ${ }^{4}$. Há dúvidas sobre quais procedimentos são mais importantes para a formação médica. Mesmo entre médicos recém-graduados e professores de faculdades há divergência sobre o tema. Os primeiros enfatizaram os procedimentos mais invasivos e de maior complexidade, enquanto os últimos, os procedimentos básicos $^{5}$.

Modelos hierárquicos de avaliação são utilizados para verificar as competências adquiridas, porém a definição do que aprender, quando aprender e como aprender deve ser bem estabelecida anteriormente, justificando os propósitos deste estudo.

\section{OBJETIVO}

Elaborar proposta sobre competências e habilidades a serem atingidas pelos alunos do curso de Medicina da Universidade Anhanguera-Uniderp, relativas à capacidade de realizar procedimentos diagnósticos e terapêuticos, em qual momento do currículo devem ser inseridas e qual o nível de complexidade pretendido em cada etapa do aprendizado.

\section{MÉTODO}

Buscando alternativas para solucionar tais questões, a Comissão de Planejamento do Curso de Medicina da Anhanguera-Uniderp elaborou um instrumento para planejamento pedagógico denominado matriz pedagógica, estruturada em eixos de objetivos educacionais. A aquisição de habilidades clínicas, onde figura o alcance de competências para execução de procedimentos médicos diagnósticos e terapêuticos, era um dos eixos de objetivos a atingir.

Em reunião com o corpo docente, essa Comissão apresentou os eixos referentes aos objetivos educacionais da matriz pedagógica. Após debate e esclarecimentos, com a anuência da maioria, foi constituída uma Comissão composta por 20 especialistas de áreas diversas (Clinica Médica, Ginecologia, Cirurgia-Geral, Pediatria, Medicina Social, Cardiologia, Urologia, Cirurgia Cabeça e Pescoço, Endocrinologia, Neurologia, Infectologia, Patologia) - denominada Comissão de Planejamento da Matriz Pedagógica - Procedimentos (CPMP) - para discutir a aquisição de habilidades e competências relativas a procedimentos diagnósticos e terapêuticos.

A CPMP realizou encontros semanais com aproximadamente três horas de duração. Os materiais utilizados foram: a matriz pedagógica - aquisição de habilidades clínicas, como diretriz para a formulação das propostas pedagógicas para a aquisição de competências relativas aos procedimentos, as Diretrizes Curriculares 2001, como fundamentação teórica para as discussões, e o Projeto Pedagógico do curso, como balizador dos encaminhamentos.

O produto da primeira etapa do trabalho foi uma lista de possíveis procedimentos médicos diagnósticos e terapêuticos, sugeridos pelos estudos e debates do grupo e que estavam em consonância com as Diretrizes Curriculares Nacionais². Seguiu-se a construção coletiva de um consenso do grupo, mediante aprovação de mais de $75 \%$ dos integrantes da comissão, quanto aos procedimentos que de fato deveriam integrar o currículo.

Concluída a primeira etapa, partiu-se para discutir o momento de inserir cada procedimento no curso de Medicina, respeitando os pressupostos do currículo integrado e da espiral do conhecimento, para, só então, definir o grau de complexidade a ser alcançado como objetivo educacional na respectiva etapa.

Verificou-se que a taxonomia de Bloom ${ }^{6}$, referência no processo de ensino-aprendizado para o desempenho cognitivo, não atendia plenamente aos quesitos para o domínio psicomotor. Para classificar os procedimentos de acordo com níveis de complexidade, a CPMP utilizou uma modificação da taxonomia de Simpson ${ }^{7}$. Esta taxonomia prevê um aprendizado progressivo no qual níveis de menor complexidade são pré-requisitos para etapas subsequentes (Tabela 1). 


\section{TABELA 1}

Taxonomia para classificação do domínio psicomotor.

\begin{tabular}{|c|c|c|}
\hline Nível & Categoria & Descrição \\
\hline 1 & Percepção & $\begin{array}{l}\text { Atenção a todos os movimentos } \\
\text { envolvidos na ação, conexões e } \\
\text { implicações. }\end{array}$ \\
\hline 2 & Posicionamento & $\begin{array}{l}\text { Colocar-se em posição correta } \\
\text { e eficiente para executar os } \\
\text { movimentos. }\end{array}$ \\
\hline 3 & $\begin{array}{l}\text { Execução } \\
\text { acompanhada }\end{array}$ & $\begin{array}{l}\text { Corretamente posicionado, executa } \\
\text { movimentos (hesitante, imperfeito, } \\
\text { parcial). Acompanhado do professor } \\
\text { ou tutor. }\end{array}$ \\
\hline 4 & $\begin{array}{l}\text { Mecanização ou } \\
\text { automatização }\end{array}$ & $\begin{array}{l}\text { Ações executadas integralmente, ciclo } \\
\text { completo de movimento, coordena a } \\
\text { ação com outras. }\end{array}$ \\
\hline 5 & $\begin{array}{l}\text { Completo domínio } \\
\text { de movimentos }\end{array}$ & $\begin{array}{l}\text { Maestria sobre as ações. É capaz de } \\
\text { ensinar. }\end{array}$ \\
\hline
\end{tabular}

\section{RESULTADOS}

\begin{tabular}{lll}
\multicolumn{2}{c}{ TA B ELA 2: } \\
Procedimentos classificados como nível 2 e sua \\
\multicolumn{1}{c}{ distribuição na matriz curricular. }
\end{tabular}

Os procedimentos foram agrupados de acordo com o nível de complexidade esperado para o acadêmico ao final do curso médico. Houve consenso em que os níveis 1, 2 e 3 sejam desenvolvidos nos quatro primeiros anos do curso, e os níveis 4 e 5, no internato. Os procedimentos foram classificados pela taxonomia referida, viabilizando o processo avaliativo.

Após esta definição, os procedimentos foram distribuídos na matriz curricular e divididos, didaticamente, em momentos de aprendizagem do domínio cognitivo (teorização) e do domínio motor propriamente dito (execução e acompanhamento). As tabelas de 2 a 5 demonstram a classificação dos procedimentos por nível de complexidade e por etapas de aprendizado.

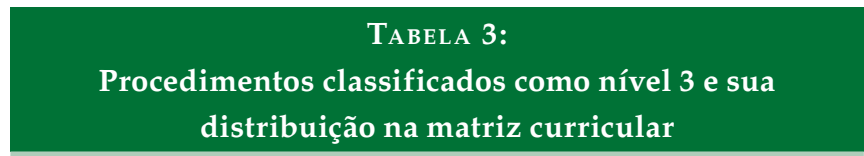

Procedimento

Paracentese

Toracocentese

Punção vesical

Cateterização venosa central

Cateterização venosa central

Bloqueios peri e raqui-medulares

Auxílio a parto cesareana

Cateterização de veia e artéria umbilicais

Punção arterial

Tala gessada

Primeiros socorros

(queimados e acidentados)

Cricotiroidostomia em modelos

Biópsia excisional

Punção articular (adultos)

Retirada de corpo estranho (nariz e ouvido)
Motor

$3^{\circ}$ ano / Internato Internato nível nível Terciário. terciário.

$4^{\circ}$ ano / Internato Internato nível nível Terciário. terciário.

$3^{\circ}$ ano / Internato Internato nível nível Terciário. terciário.

$3^{\circ}$ e $4^{\circ}$ anos / Internato nível Terciário.

Internato nível terciário.

Internato nível

$3^{\circ}$ ano / Internato nível Terciário.

Terciário / Internato regional.

$2^{\circ}$ ano - habilidades médicas / técnica cirúrgica.

Internato nível Terciário / regional.

$2^{\circ}$ ano / Internato Internato nível nível Terciário. Terciário.

$3^{\mathrm{o}}$ e $4^{\mathrm{o}}$ ano / Internato - todos.

Internato - todos.

Internato nível $4^{\mathrm{o}}$ ano / Internato nível Terciário.

Terciário / internato regional.

$2^{\mathrm{o}}$ ao $4^{\mathrm{o}}$ ano.

Internato nível primário / terciário.

$4^{\mathrm{o}}$ ano.

$4^{\mathrm{o}}$ ano / Internato Terciário.

Internato nível Internato nível Secundário. Secundário. $4^{\mathrm{o}}$ ano.

Internato nível Terciário.

Internato nível Internato nível Secundário. secundário / Terciário. 


\section{TABELA 4:}

Procedimentos classificados como nível 4 e sua distribuição na matriz curricular.

\begin{tabular}{|c|c|c|}
\hline Procedimento & Cognitivo & Motor \\
\hline $\begin{array}{l}\text { Punção intra-óssea } \\
\text { para reposição } \\
\text { volêmica }\end{array}$ & $3^{o}$ e $4^{o}$ anos. & $\begin{array}{l}4^{\circ} \text { ano / Internato } \\
\text { nível terciário. }\end{array}$ \\
\hline $\begin{array}{l}\text { Manuseio de } \\
\text { drenos: posição } \\
\text { adequada, } \\
\text { oscilação, etc }\end{array}$ & $\begin{array}{l}\text { Internato nível } \\
\text { terciário. }\end{array}$ & $\begin{array}{l}\text { Internato nível } \\
\text { terciário. }\end{array}$ \\
\hline $\begin{array}{l}\text { Lavagem gástrica e } \\
\text { clister Cateterização } \\
\text { naso-gástrica }\end{array}$ & $3^{\circ}$ e $4^{\circ}$ anos. & $\begin{array}{l}\text { Internato nível } \\
\text { terciário / } 4^{\circ} \\
\text { ano primário / } \\
\text { Habilidades Médicas } \\
3^{\circ} \text { ano. }\end{array}$ \\
\hline $\begin{array}{l}\text { Pnução lombar com } \\
\text { coleta de líquor }\end{array}$ & $\begin{array}{l}4^{\circ} \text { ano / Internato } \\
\text { nível secundário / } \\
\text { terciário. }\end{array}$ & $\begin{array}{l}\text { Internato nível } \\
\text { secundário / terciário. }\end{array}$ \\
\hline $\begin{array}{l}\text { Bloqueio anestésico } \\
\text { loco-regional e } \\
\text { troncular }\end{array}$ & $\begin{array}{l}2^{\circ} \text { e } 3^{\circ} \text { anos / } \\
\text { Internato nível } \\
\text { Terciário. }\end{array}$ & $\begin{array}{l}\text { Internato nível } \\
\text { secundário / terciário. }\end{array}$ \\
\hline Parto normal & $\begin{array}{l}2^{\circ} \text { e } 3^{\circ} \text { ano / } \\
\text { Internato nível } \\
\text { Terciário. }\end{array}$ & $\begin{array}{l}\text { Internato nível } \\
\text { Terciário / Internato } \\
\text { regional. }\end{array}$ \\
\hline $\begin{array}{l}\text { Imobilização de } \\
\text { fraturas }\end{array}$ & $\begin{array}{l}4^{\circ} \text { ano / Internato } \\
\text { nível Terciário. }\end{array}$ & $\begin{array}{l}\text { Internato nível } \\
\text { Terciário / Internato } \\
\text { regional. }\end{array}$ \\
\hline $\begin{array}{l}\text { Redução de } \\
\text { luxação e pronação } \\
\text { dolorosas (crianças) } \\
\text { (em modelos) }\end{array}$ & $4^{\mathrm{o}}$ ano. & $\begin{array}{l}\text { Internato nível } \\
\text { Terciário. }\end{array}$ \\
\hline $\begin{array}{l}\text { Remoção de poli- } \\
\text { traumatizados }\end{array}$ & $1^{\circ}, 2^{\circ}$ e $4^{\circ}$ ano. & Treinamento bonecos \\
\hline $\begin{array}{l}\text { Desobstrução } \\
\text { supra-glótica de } \\
\text { vias aéreas }\end{array}$ & $4^{\mathrm{o}}$ ano. & $\begin{array}{l}4^{\circ} \text { ano - treinamento } \\
\text { em bonecos / } \\
\text { Internato Terciário. }\end{array}$ \\
\hline $\begin{array}{l}\text { Intubação oro- } \\
\text { traqueal; mascara, } \\
\text { balão e cânula }\end{array}$ & $\begin{array}{l}1^{\circ} \text { e } 4^{\circ} \text { ano / } \\
\text { Internato nível } \\
\text { Terciário. }\end{array}$ & $\begin{array}{l}\text { Treinamento em } \\
\text { bonecos / Internato } \\
\text { nível Terciário. }\end{array}$ \\
\hline Contenção & $2^{\circ}$ e $3^{\circ}$ anos. & $\begin{array}{l}2^{\circ} \text { e } 3^{\circ} \text { anos / Internato } \\
\text { nível Terciário. }\end{array}$ \\
\hline $\begin{array}{l}\text { Limpeza e lavagem } \\
\text { de olhos }\end{array}$ & $4^{o}$ ano. & $\begin{array}{l}4^{\circ} \text { ano Internato nível } \\
\text { secundário. }\end{array}$ \\
\hline Curativos & $1^{\mathrm{o}}$ ao $4^{\mathrm{o}}$ ano & $\begin{array}{l}1^{\circ} \text { ao } 4^{\circ} \text { ano - Internato } \\
\text { Todos. }\end{array}$ \\
\hline $\begin{array}{l}\text { Debridamento } \\
\text { simples de } \\
\text { pequenas áreas }\end{array}$ & Internato Todos. & Internato \\
\hline Biópsias incisionais & $\begin{array}{l}\text { Internato nível } \\
\text { Secundário. }\end{array}$ & $\begin{array}{l}\text { Internato Nível } \\
\text { secundário. }\end{array}$ \\
\hline $\begin{array}{l}\text { Instrumentação } \\
\text { cirúrgica }\end{array}$ & $3^{o}$ e $4^{\circ}$ anos. & $\begin{array}{l}3^{\circ} \text { e } 4^{\circ} \text { anos / Internato } \\
\text { nível Terciário. }\end{array}$ \\
\hline
\end{tabular}

TABELA 5:

Procedimentos classificados como nível 5 e sua distribuição na matriz curricular.

\begin{tabular}{ccl} 
Procedimento & Cognitivo & \multicolumn{1}{c}{ Motor } \\
Cateterizção Vesical & $2 \circ$ ano / Internato. & $2^{\circ}$ ano / Internato \\
& & $\begin{array}{l}\text { Nível primário } \\
\text { / secundário / } \\
\text { terciário. }\end{array}$
\end{tabular}

Acesso venoso periférico ADULTO E CRIANÇA

Analgesia e sedação

Punção capilar; (ponta de dedo em dedo em crianças)

Aspiração de vias aéreas

RCP Básica
RCP Avançada
Caixa de Hood
Coleta de material
para exames (sangue,
urina, fezes e
secreção vaginal)

\section{REALIZAR ECG}

Preparo e fármacos

Limpeza de canal $\quad 2 \%$ ano. auditivo Drenagem de
abscessos

Limpeza de feridas e debridamento

Sutura de pele e $\quad 3^{\circ}$ e $4^{\circ}$ anos. mucosas

Assepsia, antissepsia e paramentação

$1^{\circ}$ ao $4^{\circ}$ ano Internato - primário / secundário / terciário.

Retirada de pontos (nível 5).

$3^{\circ}$ e $4^{\circ}$ anos e Internato Todos.

$1^{\mathrm{o}}$ ao $4^{\mathrm{o}}$ ano adultos e calcanhar $\mathrm{e}$ administração de $1^{\circ}$ ano (níveis 1,2 e 3) $1^{\circ}$ ano (níveis 1,2 e 3) $/ 3^{\circ}$ e $4^{\circ}$ anos (níveis / $3^{\circ}$ e $4^{\circ}$ anos (níveis 3 e 4) / Internato 3 e 4) / Internato Nível Primário e Nível Primário e Terciário (nível 5). Terciário (nível 5).

$3^{\circ}$ ano / Internato Terciário.

Internato Terciário / Internato primário.

$1^{\circ}$ ao $4^{\circ}$ ano -

Primário / Internato todos os níveis.

Internato nível Terciário.

$2^{\circ}$ ao $4^{\circ}$ / Internato Terciário.

$4^{\circ}$ ano - Internato..

$4^{\mathrm{o}}$ ano / Internato nível Terciário.

$1^{\circ}$ ao $3^{\circ}$ ano $1^{\circ}$ ano (níveis 1,2 e $\quad 1^{\circ}$ ano (níveis 1,2 e 3); $3^{\circ}$ e $4^{\circ}$ anos (níveis 3); $3^{\circ}$ e $4^{\circ}$ anos (níveis 3 e 4) / Internato 3 e 4) / Internato primário e terciário primário e terciário (nível 5). $1^{\circ}$ ano Via Oral, inalatória tópica / $2^{\circ}$ ano parenteral.

Internato Nível primário / secundário.

$3^{\mathrm{o}}$ e $4^{\mathrm{o}}$ anos /

Internato Todos.

Internato Todos.

$3^{\circ}$ e $4^{\circ}$ anos / Internato Todos.

$1^{\circ}$ ao $4^{\circ}$ ano -

Internato - primário / secundário / terciário. $1^{\circ}$ ao $4^{\circ}$ ano Internato. 


\section{DISCUSSÃO}

Apesar dos avanços tecnológicos e pedagógicos, muitos alunos terminam o curso médico com deficiências em habilidades clínicas, dentre elas os procedimentos médicos ${ }^{8,9}$. Ao longo do tempo, o aprendizado dos procedimentos médicos muitas vezes teve como premissa o "ver um", "fazer um", "ensinar um"10. No entanto, com o advento da simulação médica, da melhora da definição das habilidades e competências nas matrizes curriculares e na perspectiva de maior segurança aos pacientes, urge a necessidade de mudar este paradigma.

Competência profissional é mais que a demonstração de habilidades isoladas ${ }^{11}$. Portanto, os domínios afetivos e cognitivos devem acompanhar o ensino-aprendizado do domínio psicomotor. Caso contrário, o aluno desenvolve habilidade para realizar uma tarefa (procedimento), mas não sabe quais as indicações, contraindicações ou a melhor maneira de realizá-la. Um médico competente deve ter habilidade integrativa entre o saber, o sentir e o agir ${ }^{12}$.

Alguns estudos avaliaram quais procedimentos devem ser realizados e o grau de confiança obtido ${ }^{13,14,5}$, mas poucos definiram o nível de habilidade esperado para cada procedimento. Fazer uma ou duas vezes o procedimento não significa que o aluno tenha domínio completo da técnica esperada. Sendo assim, o presente trabalho, além de desenvolver uma matriz pedagógica que selecionou os procedimentos para a graduação médica, definiu, para cada um deles, a(s) etapa(s) de integração entre os domínios psicomotor e cognitivo e o nível de complexidade esperado. A frequência de realização dos procedimentos na prática médica diária foi considerada no momento da classificação entre os diferentes níveis de complexidade. Não podemos, por exemplo, esperar que o acadêmico realize pericardiocentese (nível 2), pois são raras as oportunidades de aprendizado durante o curso inteiro de graduação, talvez durante toda a carreira médica, mas nada impede que o estudante conheça as indicações, contraindicações, técnica de realização e treinamento simulado. Ao definirem o programa de desenvolvimento de habilidades clínicas, educadores e coordenadores de curso devem ter como preocupação inicial o reconhecimento das barreiras (epidemiológicas, legais e éticas) para a realização de procedimentos por parte dos acadêmicos de Medicina, levando em consideração a particularidade de cada instituição ou região em que está inserida.

O modelo conhecido da pirâmide de Miller organiza a competência clínica em quatro etapas: (1) sabe - relembra os fatos, teorias e princípios; (2) sabe como - habilidade em resolver problemas e descrever procedimentos; (3) mostra como - demonstra habilidade em ambiente controlado; (4) faz - realiza na prática ${ }^{15}$. Esta classificação define com mais clareza o nível de habilidade esperado para cada etapa de realização do procedimento. Assim como na música, há várias habilidades básicas a serem adquiridas antes da apresentação em público. As habilidades musicais para tocar uma nota, uma melodia e, após, a harmonia para apresentação em conjunto são análogas às habilidades de segurar o bisturi, dissecar, fazer uma anastomose e, finalmente, as habilidades de decidir, liderar e julgar ${ }^{16}$.

É fundamental que a mesma importância dada ao aprendizado de procedimentos complexos e invasivos - intubação, acesso venoso central, dissecção venosa, etc. - seja destinada ao aprimoramento das habilidades em procedimentos básicos - acesso venoso, sondagem vesical e nasogástrica, etc. Acredita-se que os estudantes atribuam menor valor aos procedimentos básicos por acreditarem que sejam atividades de responsabilidade de outras profissões, como enfermeiros ou socorristas $^{5}$. A sistematização de distribuição dos procedimentos médicos por nível de complexidade, conforme aqui proposta, pode facilitar a compreensão de docentes e discentes sobre os objetivos educacionais a serem alcançados e, por conseguinte, o desenvolvimento de métodos avaliativos eficazes.

Nos últimos anos, presenciamos a emergência do uso de simulações avançadas para ensinar procedimentos médicos, o que inclui manequins computadorizados usados para simular atendimentos de reanimação cardiopulmonar, manutenção das vias aéreas, atendimento pré-hospitalar, etc. ${ }^{17}$. Apesar da importância da discussão da eficácia dos diferentes tipos de simulação, mais relevante ainda é definir as competências necessárias e em que etapa da graduação médica devem ser desenvolvidas, aplicando-se a simulação como uma das estratégias de aprendizado. O presente estudo apresentou uma proposta de definição das competências e habilidades referentes aos procedimentos médicos a serem atingidas pelos acadêmicos de Medicina, sendo que experiências vindouras de outros autores podem contribuir para o seu aprimoramento.

\section{CONCLUSÃO}

Este estudo teve como objetivo sistematizar a distribuição das competências e habilidades referentes aos procedimentos médicos que os acadêmicos do curso de Medicina devem alcançar no decorrer de sua vida acadêmica. Assim, após o levantamento de dados, foi possível sugerir uma proposta de como inserir tais procedimentos na matriz curricular da instituição, tendo a justa medida entre o nível de conhecimento do estu- 
dante e o nível de complexidade do procedimento médico a se exigir.

\section{REFERÊNCIAS}

1. Epstein RM, Hundert EM. Defining and assessing professional competence. JAMA. 2002; 287(2): 226-35.

2. MEC 2001. Diretrizes Curriculares Nacionais do Curso de Graduação em Medicina. Retrieved Feb 28, 2011. http:// portal.mec.gov.br/cne/arquivos/pdf/CES04.pdf

3. Medical School Objectives Project. Learning objectives for medical student education-guidelines for medical schools: Report I of the Medical School Objectives Project. Acad Med. 1999; 74:13-18.

4. Sanders CW, Edwards JC, Burdenski TK. A survey of basic technical skills of medical students. Acad Med. 2004; 79:873-875.

5. Fitch MT, Kearns S, Manthey DE. Faculty physicians and new physicians disagree about which procedures are essential to learn in medical school. Med Teach 2009; 31: 342-347.

6. Bloom BS, Engelhart MD, Furst EJ, Hill WH, Krathwohl DR. 1956. Taxonomy of Educational Objectives, the classification of educational goals - Handbook I: Cognitive Domain New York: McKay

7. Simpson E. The classification of educational objectives in the psychomotor domain: The psychomotor domain. 1972; Vol. 3. Washington, DC: Gryphon House.

8. Holmboe ES. Faculty and the observation of trainees'clinical skills: problems and opportunities. Acad Med. 2004; 79(1):16-22.

9. Amaral E, Domingues RCL, Zeferino AMB. Assessing Clinical Competence:Structured Observation. Rev Bras Educ Med 2007; 31 (3) : 287 - 290.

10. Mason WT, Strike PW. See one, do one, teach one-Is this still how it works? A comparison of the medical and nursing professions in the teaching of practical procedures. Med Teach. 2003; 25:664-666.

11. Eraut M. Learning professional processes: public knowledge and personal experience. In: Eraut M, ed. Developing Professional Knowledge and Competence. London, England: Falmer Press 1994: 100-122.

12. Gale J, Marsden P. Clinical problem solving: the beginning of the process. Med Educ. 1982; 16:22-26.

13. Wu EH, Elnicki DM, Alper EJ, Bost JE, Corbett ECJr, Fagan MJ, Mechaber A, Ogden PE, Sebastian JL, Torre DM. Procedural and Interpretive Skills of Medical Students: Experiences and Attitudes ofThird-Year Students. Acad Med. 2006; 81:548-551.
14. Elnicki DM, van Londen J, Hemmer PA, Fagan M, Wong R. U.S. and Canadian internal medicine clerkship directors' opinions about teaching procedural and interpretive skills to medical students. Acad. Med. 2004 Nov;79(11):110815. Miller GE. The assessment of clinical skills/competence/performance. Acad Med. 1990; 65 Suppl 9: 63-7.

15. Windsor JA. Role of simulation in surgical education and training. ANZ J Surg. 2009; 79:27-132

16. Michelson JD, Manning L. Surgical Education Competency assessment in simulation-based procedural education. The American Journal of Surgery. 2008; 196:609-615

\section{CONTRIBUIÇÃO DOS AUTORES}

Prof. André Luís Alonso Domingos: Idealizador do trabalho, participou de todas as reuniões que escolheram e classificaram os procedimentos. Participou da construção do texto e envio à revista. Gabriel Costa Almeida: Participou da elaboração do texto, da realização da discussão dos artigos. Prof ${ }^{-}$Sandra C. dos Santos: Idealizadora do trabalho, participou de todas as reuniões que escolheram e classificaram os procedimentos. Participou da revisão do trabalho e da discussão dos artigos. Prof $^{\mathrm{a}}$ Márcia Maria Silva: participou de todas as reuniões que escolheram e classificaram os procedimentos. Pesquisou todas as taxonomias relacionadas aos procedimentos médicos e aquisição de habilidades. Participou da discussão do trabalho. Prof. Antonio Carlos Carbonaro Salles: Participou de todas as reuniões que escolheram e classificaram os procedimentos. Participou da organização das tabelas e revisão do texto. Acadêmico Alexandre de Abreu Lima: Participou da elaboração do texto, levantamento bibliográfico, normas de publicação e discussão dos artigos.

\section{CONFLITO DE INTERESSES}

Não há conflito de interesses.

\section{ENDEREÇO PARA CORRESPONDÊNCIA}

André Luís Alonso Domingos

Rua Sete de Setembro, 2262, apto 802.

Centro - Campo Grande

CEP 79020310 MS

andre.domingos@uniderp.edu.br 